\title{
Práticas educativas em um centro de atenção psicossocial álcool e drogas: percepção dos usuários
}

\author{
Educational practices in a psychosocial care center alcohol and drugs: user perception \\ Prácticas educativas en un centro de atención psicosocial alcohol y drogas: percepción \\ del usuario
}

Lilia Magno de Abreu Teixeira ${ }^{1 *}$, Xaene Maria Fernandes Duarte Mendonça1, Thaís de Oliveira Carvalho Granado Santos ${ }^{1}$, Barbara Liliane Cardoso Costa ${ }^{1}$, Ana Clara Barros de Sousa ${ }^{1}$, Giovana Sousa dos Santos ${ }^{1}$, Luna Carolina Cardoso Castro², Claudia Daniele Tavares Dutra ${ }^{1}$, Patrícia Miranda Mendes ${ }^{1}$.

\section{RESUMO}

Objetivo: Analisar a percepção dos usuários de um Centro de Atenção Psicossocial Álcool e Drogas (CAPSAD) quanto à importância das atividades educativas promovidas pela equipe de nutrição. Métodos: Trata-se de um estudo de delineamento descritivo e exploratório, com abordagem quanti-qualitativa, desenvolvido no Pará, no período de junho a outubro/2019, em um CAPSAD, no qual foi aplicado formulário contendo questões sobre a avaliação das atividades educativas e sobre a percepção dos usuários a respeito dessas práticas. Resultados: Participaram do estudo 23 usuários, sendo que todos consideraram as atividades educativas importantes, avaliando-as em ótima (52,1\%) e boa $(47,8 \%)$, e $91,3 \%$ acredita que as atividades contribuem com o tratamento realizado no CAPSAD. Na análise qualitativa observou-se que a maioria das falas associou a importância das atividades educativas à aquisição de novos conhecimentos, e por ser uma forma de distanciar ou reduzir os pensamentos e sentimentos provenientes da dependência da droga. Conclusão: As atividades educativas desenvolvidas no CAPSAD além de auxiliar na promoção da saúde, colaboram com o aprendizado e com o tratamento terapêutico dos usuários. Em relação à percepção evidenciou-se que a importância das práticas educativas esteve relacionada ao aprendizado e por ser uma forma de ocupar a mente e afastá-los das drogas.

Palavras-chave: Atividades educativas, Saúde mental, Ações terapêuticas.

\begin{abstract}
Objective: Analyze the perception of users of a Psychosocial Care Center for Alcohol and Drugs regarding the importance of educational activities promoted by the nutrition team. Methods: This is a descriptive and exploratory study, with quantitative and qualitative approach, developed in Pará, from June to October 2019, in which was applied a form containing questions about the evaluation of educational activities and the users' perception about these practices. Results: 23 users participated in the study and all considered the educational activities important, evaluating them as excellent $(52.1 \%)$ and good (47.8\%), and $91.3 \%$ believe the activities contribute with the treatment performed in the Center. In the qualitative analyses, the majority of the statements associated the importance of educational activities with the acquisition of new knowledge and means to distance or reduce the thoughts and feelings derived from drug addiction. Conclusion: The educational activities developed in the service, besides helping in health promotion, collaborate with users' learning and therapeutic treatment. Regarding perception, it was evident that the importance of educational practices was related to learning and as a way of occupying the mind and getting away from drugs.
\end{abstract}

Keywords: Educational activities, Mental health, Therapeutic actions.

1 Universidade Federal do Pará (UFPA), Belém - PA. *E-mail: liliamagno24@gmail.com

${ }^{2}$ Secretaria Municipal de Saúde do Pará (SESMA), Belém - PA. 


\section{RESUMEN}

Objetivo: Analice la percepción de los usuarios de un Centro de Atención Psicosocial para el Alcohol y las Drogas con respecto a la importancia de las actividades educativas promovidas por el equipo de nutrición. Métodos: Este es un estudio descriptivo y exploratorio, com enfoque cuantitativo y cualitativo, desarrollado en Pará, de junio a octubre de 2019, en el que se aplicó um formulario que contiene preguntas sobre la evaluación de actividades educativas y la percepción de los usuários sobre estas prácticas. Resultados: 23 usuarios participaron en el estudio, y todos consideraron importantes las actividades educativas, calificándolas como excelentes $(52.1 \%)$ y buenas $(47.8 \%)$, y $91.3 \%$ creen que las atividades contribuyen con el tratamiento realizado en el Centro. En los análisis cualitativo, se observo que lamayoría de las declaraciones asociado la importancia de las actividades educativas con la adquisición de nuevos conocimientos y um medios para distanciar o reducir los pensamientos y sentimientos derivados de la adicción a las drogas. Conclusión: Las atividades educativas desarrolhadas en lo Centro, además de ayudar en la promoción de la salud, colaboran com el aprendizaje y el tratamento terapéutico de los usuários. Com respecto a la percepción, era evidente que la importancia de las prácticas educativas estaba relacionada con el aprendizaje y com una forma de ocupar la mente y alejarse de las drogas.

Palabras clave: Actividades educativas, Salud mental, Acciones terapéuticas.

\section{INTRODUÇÃO}

Por muito tempo a Política de Saúde do Brasil era omissa às questões que envolviam a prevenção e tratamento de transtornos decorrentes do uso de álcool e outras drogas. Foi somente no ano de 2002 que este tema foi considerado um problema de saúde pública, e instituiu-se o Programa Nacional de Atenção Comunitária Integrada aos usúarios de álcool e outras drogas, que é articulado à Rede de Atenção Psicossocial (RAPS)(BRASIL, 2005).

Neste contexto surgem os Centros de Atenção Psicossocial Álcool e Drogas (CAPS AD), pontos estratégicos da RAPS, que atendem indivíduos em dependência e/ou uso abusivo de álcool e drogas (BRASIL, 2015). A Política de Saúde Mental preconiza que estes são serviços abertos, comunitários, pautados na perspectiva da construção dos projetos terapêuticos singulares, que devem ser compartilhados entre: usuário, familia e equipe, visando o cuidado integral dos indivíduos (DUTRA VFD, et al., 2017).

Dentre os diversos serviços ofertados no CAPS AD, estão as atividades em grupo que visam promover a sociabilidade, trabalhar as dificuldades de relacionamentos, promover a troca de experiências e afetos, desenvolver a autoestima e autonomia (BRASIL, 2015). Atualmente, a educação em saúde é bastante presente no âmbito da saúde mental, por meio de diferentes atividades como: oficina culinária, oficina terapêutica, e grupo educativo (SCHLICKMANN DS, et al., 2016; STEILL EPS, et al., 2017; ARAÚJO AK e SOARES VL, 2018).

De acordo com Brasil (2007), a educação em saúde é uma extensão das práticas educativas, pois envolve um processo de trabalho em que se almeja transformar algo existente em novo, seja uma ideia, mentalidade ou valor. E para que as ações educativas sejam eficazes é necessário que se avalie os resultados individuais e coletivos, e se estabeleça relação entre os problemas de saúde de determinado grupo e sua condição de vida, considerando assim sua realidade.

As práticas educativas têm o objetivo de desenvolver o pensamento crítico e reflexivo nos individuos de forma que sejam capazes de opinar nas decisões de saúde que envolvem o cuidado de si ou do coletivo. (FALKENBERG MB, et al., 2014).

Almeida ER, et al. (2014) afirmam que a educação é capaz de promover mudanças no estilo de vida de uma pessoa. Porém, Freire (2011, apud ALMEIDA ER, et al. 2014) conclui que não bastar apenas aprender algo novo, é necessário que o indivíduo reflita e tenha ações que o direcione a um novo modo de vida.

Logo, quando participamos do processo de cuidado à pessoa em sofrimento psíquico ou transtorno mental, é importante que se reconheça e respeite as escolhas que envolvem seu tratamento, pois é uma forma do indivíduo exercer autonomia em questões que de saúde (CARDOSO MPO, et al., 2016). 
Diante disso, é de suma importância conhecer a percepção dos usuários do serviço a respeito das atividades educativas desenvolvidas no CAPS AD, uma vez que sua opinião exerce papel importante em seu tratamento e aprendizado; e por existirem diversas atividades de educação em saúde sendo elaboradas na area de saúde mental.

Por isso, busca-se atráves deste estudo analisar a percepção dos usuários de um CAPS AD quanto a importância das atividades educativas promovidas pela equipe de nutrição. Para tanto, foram definidos os seguintes objetivos especificos: identificar o perfil sociodemográfico dos participantes, conhecer a opinião dos usuários em relação as atividades educativas; avaliar as práticas educativas como promotoras de novos conhecimentos e identificar as possíveis contribuições dessas ações educativas para o tratamento dos usuários.

\section{MÉTODOS}

Estudo de delineamento descritivo e exploratório, com abordagem quanti-qualitativa, desenvolvido em um município do estado do Pará, no período de junho a outubro de 2019. Participaram da pesquisa usuários de um Centro de Atenção Psicossocial Álcool e outras Drogas (CAPS AD), compondo a amostra calculada considerando-se o número de novos acolhimentos mensais, considerando-se nível de confiança de 95\%.

Os critérios de inclusão para participação na pesquisa foram: ser usuário do CAPS AD, participar das atividades educativas promovidas pela equipe de nutrição, ter idade superior a 18 anos; ser capaz de dar respostas atendendo aos objetivos do estudo, e assinar previamente o Termo de Consentimento Livre e Esclarecido. Os critérios de exclusão foram: está sob efeito de alguma substância psicoativa, ou já ter respondido as questões da pesquisa em outro momento.

Foram consideradas para esta pesquisa as atividades educativas de Nutrição de caráter aberto, que comumente já ocorrem no CAPS AD. Na elaboração e planejamento das atividades considerou-se as datas do Calendário da Saúde, e, de acordo, com o tema foram construídas, sendo realizadas por meio de roda de conversa, oficina culinária, horta, jogos educativos, e dinâmicas. Em todas as atividades os usuários eram estimulados a participar e expor suas opiniões e dúvidas. Ao final de cada atividade os usuários eram convidados a participar da pesquisa, sendo que poderiam responder ao formulário somente uma vez, independente da participação em outras atividades.

Definiu-se como instrumento de coleta de dados um formulário com perguntas abertas e fechadas, sendo as entrevistas registradas por meio da gravação de áudio, conforme consentimento prévio do entrevistado. Para conhecer o perfil dos participantes da pesquisa foram coletados dados sociodemográficos (idade, sexo, grau de escolaridade, estado civil, renda, e situação de moradia). Em relação as atividades educativas, foram realizadas perguntas referentes a participação, a importância da atividade, o aprendizado, a classificação da atividade, e a contribuição para o tratamento.

As entrevistas foram realizadas no turno da tarde, por adequação ao horário de trabalho da equipe. As falas foram gravadas e transcritas na íntegra para posterior análise. De posse do conteúdo para a realização da pesquisa, os dados foram tabulados no programa Microsoft Office Excel 2016. A análise dos discursos foi realizada com auxílio da Plataforma Interface de R pourles Analyses Multidimensionnelles de Textes et de Questionnaires (IRAMUTEQ), um software gratuito que possibilita vários tipos de análises estatísticas de dados textuais.

A análise utilizada foi a nuvem de palavras, que possibilita com que as palavras mais mencionadas se agrupem graficamente de acordo com sua frequência, sendo possível identificar as palavras-chaves no corpus, ou seja, no texto formado por todas falas dos entrevistados (KAMI MTM, et al., 2016). Para melhor compreensão dos resultados e garantindo o sigilo dos participantes, as falas obtidas nas entrevistas e mencionadas no estudo, apresentam a sigla $\mathrm{P}$ (participante) e o número de identificação dos entrevistados.

A presente pesquisa foi avaliada e autorizada pelo Núcleo de Educação Permanente da Secretaria Municipal de Saúde e posteriormente encaminhada para apreciação do Comitê de Ética em Pesquisa, sendo aprovado sob $\mathrm{n}^{\circ}$ CAAE 92460218.0.3001.0018, parecer $\mathrm{n}^{\circ} 3.592 .461$ e $\mathrm{n}^{\circ}$ CAAE: 92460218000005171 , parecer $n^{\circ} 3.046 .597$. 


\section{RESULTADOS E DISCUSSÕES}

Foram avaliadas 10 atividades educativas que trabalharam temas referentes ao calendário do Ministério da Saúde, como pode ser observado (Quadro 1).

Quadro 1 - Atividades educativas avaliadas no estudo.

\begin{tabular}{|c|c|c|}
\hline & Temas & Atividades educativas \\
\hline 1 & Dia Mundial do Meio Ambiente & $\begin{array}{l}\text { Roda de conversa e jogo "Alimento in natura e sua } \\
\text { cor" }\end{array}$ \\
\hline 2 & Dia Nacional de Combate ao Fumo & Roda de conversa e dinâmica "O que posso fazer?" \\
\hline 3 & Dia do Nutricionista & $\begin{array}{l}\text { Oficina culinária e dinâmica "O que é a nutrição } \\
\text { para você?" }\end{array}$ \\
\hline 4 & Dia Mundial de Prevenção ao Suicídio & Roda de conversa e cultivo da horta \\
\hline 5 & Aniversário do SUS & $\begin{array}{l}\text { Oficina culinária e apresentação teatral } \\
\text { "InformaSUS" }\end{array}$ \\
\hline 6 & Dia Mundial de Combate ao Estresse & Técnicas de relaxamento e cultivo da horta \\
\hline 7 & Dia Mundial da Alimentação & Oficina culinária e caça-palavras "sustentável" \\
\hline 8 & Dia Nacional de Prevenção da Obesidade & $\begin{array}{l}\text { Jogo "Quais as causas da obesidade? "e Jogo de } \\
\text { tabuleiro. }\end{array}$ \\
\hline 9 & Dia Internacional da Saúde mental & Bingo das frutas regionais \\
\hline 10 & Campanha Outubro Rosa & $\begin{array}{c}\text { Oficina culinária e dinâmica "Cada corpo uma } \\
\text { história" }\end{array}$ \\
\hline
\end{tabular}

Fonte: Teixeira LMA, et al., 2020.

Participaram da pesquisa 23 usuários do serviço, sendo a maioria do sexo masculino $(82,6 \%)$, na faixa etária de 30 a 45 anos (47,8\%), solteiros (69,5\%). Em relação a escolaridade, prevaleceu o nível médio $(47,8 \%)$. A presença do desemprego foi predominante $(69,5 \%)$, prevalecendo entre os entrevistados renda familiar de até 2 salários mínimos (SM), com percentual equivalente a $52,1 \%$, quando somados os que vivem com menos de um salário e até dois salários.

Quanto à moradia, mais da metade referiu residir em casa própria, alugada ou cedida $(69,5 \%)$, porém, é possível observar um percentual significativo e igual de usuários em situação de rua $(21,7 \%)$ e sem renda familiar (21,7\%) (Tabela 1). 
Tabela 1 - Perfil sociodemográfico dos participantes do estudo, Belém - PA, 2019.

\begin{tabular}{|c|c|c|}
\hline Características & $\mathbf{N}$ & $\%$ \\
\hline \multicolumn{3}{|l|}{ Gênero } \\
\hline Feminino & 4 & 17,3 \\
\hline Masculino & 19 & 82,6 \\
\hline \multicolumn{3}{|l|}{ Faixa etária (anos) } \\
\hline 30 a 45 & 11 & 47,8 \\
\hline 46 a 60 & 9 & 39,1 \\
\hline$>60$ & 3 & 13,0 \\
\hline \multicolumn{3}{|l|}{ Escolaridade } \\
\hline E. Fundamental & 7 & 30,4 \\
\hline E. Médio & 11 & 47,8 \\
\hline E. Superior & 5 & 21,7 \\
\hline \multicolumn{3}{|l|}{ Estado civil } \\
\hline Solteiro & 16 & 69,5 \\
\hline Casado/União Estável & 4 & 17,3 \\
\hline Divorciado & 1 & 4,3 \\
\hline Viúvo & 2 & 8,7 \\
\hline \multicolumn{3}{|l|}{ Trabalho remunerado } \\
\hline Sim & 7 & 30,4 \\
\hline Não & 16 & 69,5 \\
\hline \multicolumn{3}{|l|}{ Renda familiar } \\
\hline$<1 \mathrm{SM}$ & 6 & 26,0 \\
\hline 1 a 2 SM & 6 & 26,0 \\
\hline$>2 \mathrm{SM}$ & 2 & 8,7 \\
\hline Não sabe informar & 4 & 17,3 \\
\hline \multicolumn{3}{|l|}{ Situação de moradia } \\
\hline Sem Renda & 5 & 21,7 \\
\hline Casa própria, alugada ou cedida & 16 & 69,5 \\
\hline Abrigo & 2 & 8,7 \\
\hline Situação de rua & 5 & 21,7 \\
\hline Total & 23 & 100,0 \\
\hline
\end{tabular}

Fonte: Teixeira LMA, et al., 2020.

Os dados apresentados na Tabela 1 se assemelham aos encontrados por Bosque RM, et al. (2017), que avaliando a satisfação dos usuários de um CAPS AD, concluíram que dos 60 participantes 83,3\% pertenciam ao sexo masculino, com idade entre 31 e 40 anos (38,3\%), 43,3\% solteiros.

Assim como o estudo de Silva e Silva DL, et al. (2017), que avaliou 213 prontuários dos usuários do CAPS AD modalidade III do município de Campinas-São Paulo, e encontrou na amostra $82,6 \%$ do público sendo homens, dos 208 prontuários com informação sobre o estado civil 42,7\% eram solteiros, e de 202 que apresentavam a escolaridade 7,4 \% tinham ensino médio completo e $39,1 \%$ incompleto.

Oliveira VC, et al. (2017) quando avaliaram os 706 prontuários de usuários de um CAPD AD, além da prevalência de homens $(92,0 \%)$, solteiros $(46,0 \%)$, com renda familiar de 1 a 3 salários mínimos (50,6\%), encontrou predomínio de desemprego $(45,4 \%)$. A prevalência do sexo masculino pode estar associada a maior adesão ao tratamento nesta faixa etária (PEIXOTO C, et al., 2010). 
Este público é o que mais sofre com problemas relacionados a substâncias psicoativas, sendo que as mulheres, mesmo quando necessitam, procuram menos este tipo de serviço por sofrerem estigmas sociais (OLIVEIRA VC, et al., 2017; SILVA E SILVA DL, et al., 2017).

No que concerne à escolaridade, percebe-se que o nível de ensino neste público se difere de outros estudos que avaliaram a mesma variável, como aponta Almeida RA, et al. (2014) e Oliveira VC, et al. (2017) em que foi comum a baixa escolaridade neste público.

Em relação à participação nas atividades, 65,2\% dos 23 entrevistados afirmaram ter participado integralmente das atividades educativas, todos consideraram importantes as atividades desenvolvidas pela equipe de Nutrição (100,0\%), 52,1\% classificaram as atividades educativas em ótima e 47,8\% em boa, não havendo avaliação negativa por parte dos usuários para essa questão. No que se refere ao aprendizado, $91,3 \%$ afirmou ter aprendido algo novo durante as ações educativas e o mesmo percentual (91,3\%) foi observado para os que afirmaram que as atividades educativas contribuem para o tratamento realizado no CAPS AD (Tabela 2).

Tabela 2 - Avaliação das atividades educativas pelos usuários do CAPS AD, Belém - PA, 2019.

\begin{tabular}{ccc}
\hline Itens Avaliados & $\mathbf{N}$ & $\%$ \\
\hline Como foi sua participação na atividade? & 15 & 65,2 \\
Integral & 8 & 34,7 \\
Parcial & & \\
\hline Considera a atividade importante? & 23 & 100,0 \\
São & 0 & 0,0 \\
\hline Aprendeu algo novo? & & \\
\hline Sim & 21 & 91,3 \\
Não & 2 & 8,7 \\
\hline Como avalia a atividade? & & 52,1 \\
Ótima & 12 & 47,8 \\
Boa & 11 & 0,0 \\
Regular & 0 & 0,0 \\
\hline Ruim & 0 & 91,3 \\
\hline Sim & & 4,3 \\
Não & 21 \\
Não sabe & 1 & 4,3 \\
\hline Total & 1 & 100,0 \\
\hline A atividade pode contribuir com o tratamento? & 23 \\
\hline
\end{tabular}

Fonte: Teixeira LMA, et al., 2020.

A Reforma Psiquiátrica convida os profissionais a repensarem seu modo de cuidar, sendo necessário assim descobrir e construir práticas que promovam a participação, autonomia e reflexão dos usuários (VELÔSO TMG e EULÀLIO MC, 2016). Diante disso, acredita-se que as ações educativas foram avaliadas de forma positiva pelos usuários, pois contou com a participação integral da maioria dos participantes.

Pfluck NAD (2011) em seu estudo, quando avaliou um grupo educativo em um CAPS AD, concluiu que, através da participação ativa nas práticas educativas em saúde, poderia promover reflexão e pensamento crítico nos usuários. Compreende-se dessa forma que as novas práticas na área de saúde mental têm sido vista de maneira benéfica pelos usuários do serviço.

Entende-se que o trabalho desenvolvido juntamente com os participantes deste estudo, conseguiu alcançar os objetivos propostos aos profissionais da área de saúde mental. Promovendo assim, a participação, aprendizado e reflexão sobre questões que envolvem seu tratamento. Além disso, possibilitou 
conhecer o valor atribuído ao trabalho desenvolvido com o grupo, como pode ser observado nos resultados relacionados a percepção dos usuários sobre as atividades.

Para a análise qualitativa sobre a percepção dos usuários em relação as práticas educativas, utilizou-se três perguntas abertas, as quais foram feitas somente aqueles que responderam "sim" para as questões: "Considera a atividade importante? "; "Aprendeu algo novo?", "A atividade pode contribuir com seu tratamento? ". Para isso complementou-se as perguntas com as seguintes frases: "Por que? " aprendeu? " e "De que forma?", respectivamente.

De posse desses discursos, analisou-se a frequência das palavras mais mencionadas utilizando-se a técnica de Nuvem de Palavras do IRAMUTEQ. Com base nessas palavras, a plataforma gerou uma imagem para as respostas à questão "Por que as atividades educativas são importantes? ", sendo mais frequentes os termos gente, muito, aprender, saber, ajuda e importante (Figura 1).

Figura 1 - Nuvem de Palavras formada pelo IRAMUTEQ a partir das respostas à pergunta "Por que as atividades educativas são importantes? ", Belém - PA, 2019

\section{drogaatividade bomimportante ajuda aprender

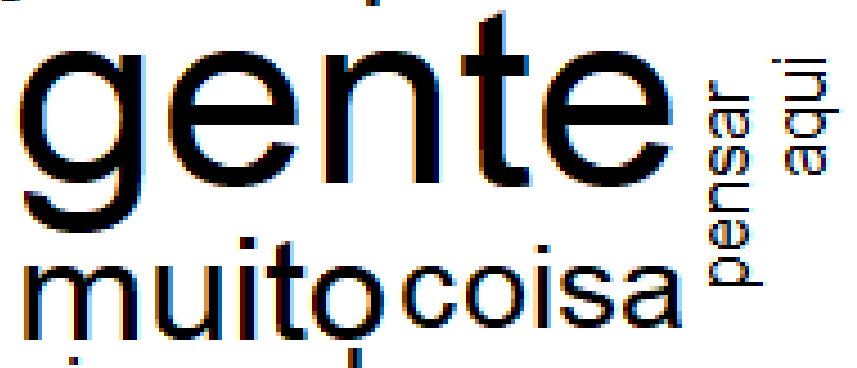 mais saber doença achar mente ficar}

Fonte: Análise textual realizada pelo IRAMUTEQ, Belém-PA, 2020. Teixeira LMA, et al., 2020.

Observa-se através da Figura 1 que foi mais frequente a palavra gente, a qual foi muito utilizada pelos usuários para retratar o coletivo, as dificuldades e necessidades do grupo. Mostrando assim, que eles acreditam que as atividades são importantes não somente para si, mas também para o grupo. Compreendese ainda, que a relevância das atividades educativas esteve relacionada ao conhecimento adquirido pela vivência com o grupo e por ser uma atividade que ajuda a afastá-los do uso e de pensamentos decorrentes da dependência do álcool e outras drogas, como pode ser observado nas falas dos entrevistados:

"Pra mim é muito importante essas atividades por causa que a gente, pra ter mais pensamentos de não ficar pensando muito em besteiras, ter um motivo de fazer comida, ou fazer umas brincadeiras. Por que essa a droga mexe muito com nosso, com nosso, mentalidade, a gente pensa só nisso, só nisso, em droga. Mas tendo esse trabalho que vocês fazem é muito importante pra nós, que a gente já alivia mais aquela coisa, aquela ansiedade, por isso eu acho mesmo muito importante o trabalho de vocês."(P7) 
"Até porque né, nutrição né, é uma coisa legal né, pra nós podermos aprender mais né sobre as coisas alimentares, o que é bom pro nosso organismo né, o nosso bem social, viver né, o bem dos alimentos, porque muitas vezes nós comemos e não sabe nem o que a gente come né, sabe o que é bom pra saúde, o que é mau pra saúde, o que irrita né, a doença né, que chama a doença pra nós né, e eu acho muito importante sim esse tipo de trabalho dentro do CAPS."(P8)

"Porque pra justamente pra desenvolver o trabalho que a gente tá querendo alcançar aqui né, de buscar inspiração pra poder justamente tratar dos problemas que a gente tem."(P15)

"É porque pra gente que é usuário ajuda muito porque ocupa nossa mente, tipo assim desvia o meu pensamento daquela coisa que eu ia fazer, vamos dizer assim, entendeu?"(P17)

Ao analisar os termos mencionados com maior frequência para a pergunta "o que você aprendeu?", obteve-se as palavras fazer, aprender, ser, como mais frequentes. Infere-se através dos termos mais mencionados que as atividades educativas promovidas pela equipe de nutrição se mostraram eficazes em promover novos conhecimentos aos participantes, sendo possível observar que mencionaram em suas falas os temas que foram abordados nas atividades, como: bolo, fruta, saúde, semente, dentre outros (Figura 2).

Figura 2 - Nuvem de Palavras constituída a partir do corpus formado pelas respostas dos usuários do CAPS AD à pergunta "O que você aprendeu?", Belém - PA, 2019.

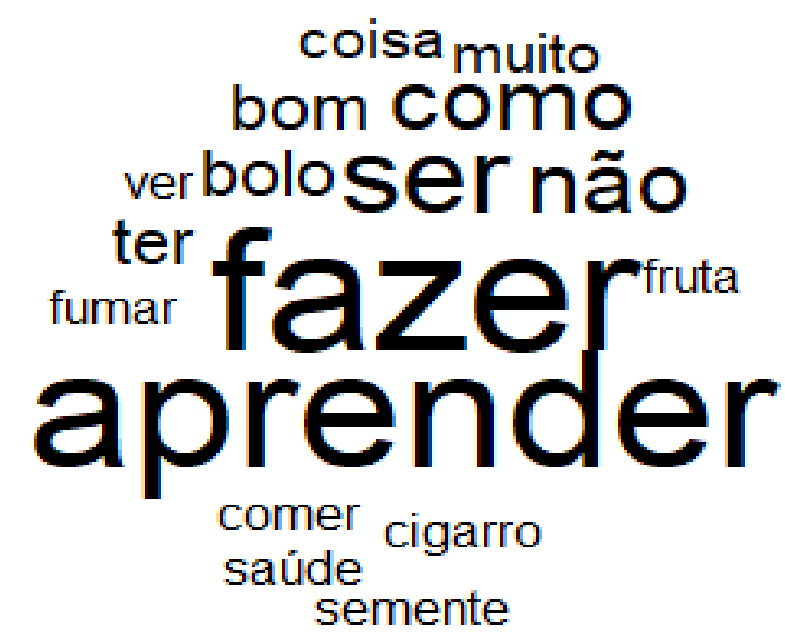

Fonte: Análise textual realizada pelo IRAMUTEQ, Belém-PA, 2020. Teixeira LMA, et al., 2020.

De acordo com a Figura 2 e os discursos que se seguem, constatou-se que os novos conhecimentos estiveram associados aos temas abordados nas práticas educativas desenvolvidas pelo grupo de nutrição, indicando-se assim que o usuário teve autonomia e participação ativa no processo de ensinoaprendizagem, além oportunizar novas experiências e aprendizados, o que pode ser confirmado através das falas a seguir:

"Novas frutas e que podemos fazer o consumo sustentável mudando nossos hábitos."(P1)

"Os benefícios de parar de fumar."(P5)

"O que eu aprendi por exemplo hoje, fazer uma pizza de jambú e couve que eu não sabia fazer né."(P8)

"Aprendi sobre as sementes que são tão pequenas, que eu não sabia que até que tinha semente essas folhas lá."(P9) 
"Aprendi a passar o óleo na forminha, vi lá quando o rapaz tava misturando as coisas, aprendi quase um pouco."(P13)

"A questão de fazer bolo né, que eu nunca tinha feito e nunca tinha visto fazer."(P17)

A Nuvem de Palavras construída pelo IRAMUTEQ, a partir das respostas dos participantes à pergunta "De que forma você acha que a atividade pode contribuir com o seu tratamento?", destaca as palavras mais mencionadas nas entrevistas realizadas, sendo as mais recorrentes ter, fazer, estar, ajuda, mente (Figura 3).

Figura 3 - Nuvem de Palavras gerada a partir dos discursos referentes à questão "De que forma você acha que a atividade pode contribuir com o seu tratamento?", Belém- PA, 2019.

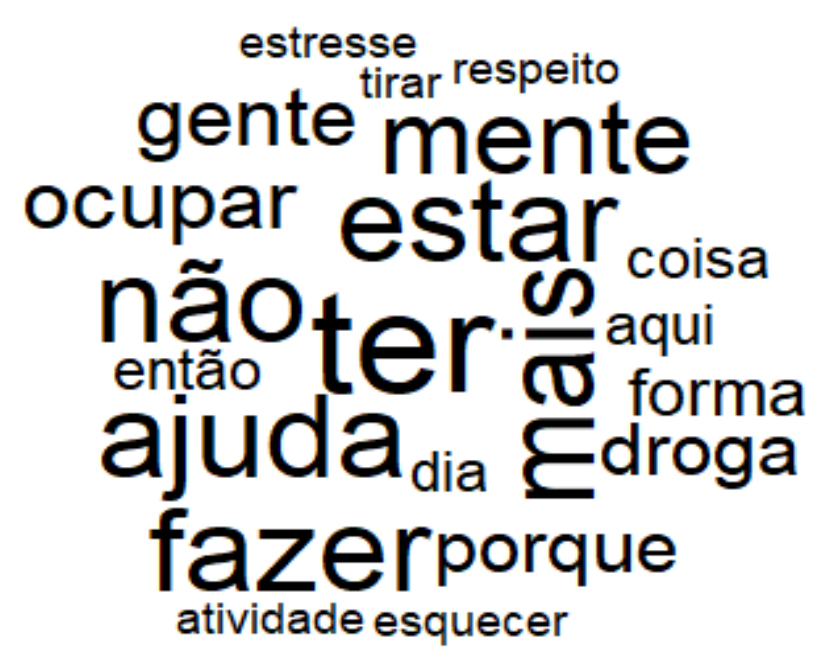

Fonte: Análise textual realizada pelo IRAMUTEQ, Belém-PA, 2020. Teixeira LMA, et al., 2020.

Confirma-se através da análise que a maioria dos usuários acredita que as atividades educativas podem auxiliar no tratamento por ser uma forma de evitar que fiquem ociosos, "ocupando a mente" com outros assuntos e atividades que os distancie ou reduzam os pensamentos e sentimentos provenientes da dependência. Além de contribuir para efetivação do tratamento, como pode ser observado nos discursos.

"Há... pelo menos a gente tá ocupando a nossa mente aqui né, a gente não tá com outros pensamentos. A gente ta, no meu caso né, eu acho que todo mundo também aqui, porque se a gente não tivesse aqui ocupando nossa mente a gente tava com..., fazendo outras coisas que não deveria fazer."(P13).

"Porque tira o estresse né, tira o estresse da gente. Por exemplo, se eu não tivesse aqui tava na mesa do bar bebendo, fumando cigarro, entendeu? Já contribui alguma coisa, se tivesse todo dia era bom né."(P14).

"Porque ela de uma maneira, ela ocupa a mente do usuário. Porque tipo assim, se a pessoa é aquele usuário que usa droga todo dia, ai ela começa a participar de uma atividade diferente, ai ela vai, começa a sentir aquela vontade de querer mudar mesmo, sabe? Por quê? Porque a mente ela tá presa naquele ciclo de todo dia, entendeu? Naquela atividade todo dia de correr atrás, de fazer o corre e usar a droga."(P17).

"De forma que faz esquecer o vício, as pessoas que consomem, mudar os lugares que frequentava. Então tudo que ocupar minha mente de forma produtiva para mim é ótimo, me faz esquecer isso."(P22). 
Evidenciou-se nos discursos dos usuários do serviço que as atividades educativas realizadas pela equipe de nutrição no CAPS AD apresentaram resultado positivo para o tratamento, proporcionando a eles bem-estar, e oportunizando momentos de socialização e possível mudança de comportamento.

Silva PPC, et al. (2019) encontrou falas semelhantes quando buscou conhecer a percepção dos usuários de um CAPS AD sobre as práticas corporais desenvolvidas. Obteve como resultado que as atividades os distraiam e distanciavam das drogas, oportunizando também um momento de socialização.

Mesmo sendo atividades distintas percebe-se que pessoas em tratamento de dependência de substâncias psicoativas demonstram a necessidade de atividades que preencham sua rotina, não os deixando ociosos, considerando assim que sua mente precisa estar ocupada para evitar pensamentos que os levem ao uso das drogas.

Resultado semelhante foi encontrado por Almeida RBF, et al. (2018) quando pesquisou sobre a percepção de usuários de crack em relação ao tratamento da dependência, os participantes relataram precisar "ocupar a mente", destacando assim a necessidade de atividades que os auxiliem a afastá-los dos pensamentos nocivos que os levam ao uso da droga.

Cruz MP, et al. (2016), quando buscou conhecer a percepção dos familiares de usuário de um CAPS AD sobre as atividades e oficinas desenvolvidas, encontraram como resultado que estas eram primordiais para o tratamento dos usuários.

Acredita-se que as práticas educativas desenvolvidas no CAPS AD, além de promover a educação em saúde, contribuem para o processo de ressocialização. E que as atividades quando elaboradas respeitando a participação e necessidade dos usuários, podem ser benéficas para o tratamento.

Porém sabe-se que estas práticas por mais que sejam bem elaboradas e aceitas pelos usuários de um CAPS AD, não são o bastante para considerá-las em si uma solução. Pois, segundo Velôso TMG e Eulálio MC (2016) somente os serviços ofertados pelas instituições são insuficientes para mudar a relação do usuário com as drogas, pois o tratamento envolve diversas questões como: afetividade, política, saúde, educação, trabalho, renda, lazer dentre outros.

Fatores esses que uma instituição por si só não consegue abarcar sozinha, mas quando bem articulada com os outros serviços por meio da RAPS, do Sistema Único de Assistência Social (SUAS) e Sistema Único de Saúde (SUS) possibilita aos usuários que outras demandas sejam supridas.

Ademais, Almeida RBF, et al. (2018) encontrou nos discursos dos participantes de sua pesquisa que, para que o tratamento tenha aspecto positivo na vida do usuário, é necessário que a pessoa decida e tenha força de vontade em permanecer no tratamento, afirmando dessa forma, a importância da coparticipação no tratamento. Os autores concluem ainda que é importante a diversificação de atividades ofertadas aos usuários, além das terapêuticas.

O estudo evidenciou que as atividades promovidas pela equipe de nutrição são importantes para os usuários. Porém, é importante conhecer a percepção de um número maior de usuários em relação a este tema. Além disso, é necessário buscar identificar os fatores que levam a não permanência dos usuários no grupo. Já que os mesmos reconhecem as práticas educativas como benéficas para seu tratamento.

\section{CONCLUSÃO}

Conclui-se que as atividades educativas desenvolvidas no CAPS AD, de acordo com a perspectiva dos usuários, podem ter ação terapêutica para indivíduos em tratamento de dependência de substâncias psicoativas e que a Nutrição desempenha papel importante neste processo. Além disso, percebe-se que os usuários valorizam as práticas educativas e conseguem apontar os benefícios adquiridos por elas. Porém, mesmo que reconheçam os benefícios, ainda se percebe que há dificuldades dos usuários em manter-se nas atividades educativas e no tratamento. Ademais, os resultados do estudo direcionam como o profissional nutricionista pode estar presente e colaborar com a equipe multiprofissional da área de saúde mental. 


\section{REFERÊNCIAS}

1. ALMEIDA ER, et al. A prática da educação em saúde na percepção dos usuários hipertensos e diabéticos. Revista Saúde Debate, 2014; 38 (101): 328-337.

2. ALMEIDA RA, et al. Perfil dos usuários de substâncias psicoativas de João Pessoa. Revista Saúde Debate, 2014; 38 (102): 526-538.

3. ALMEIDA RBF, et al. O tratamento da dependência na perspectiva das pessoas que fazem uso de crack. Revista Interface Comunicação, saúde e educação, 2018; 22 (66): 745-756.

4. ARAÚJO AK, SOARES VL. Trabalho e saúde mental: relato de experiência em um CAPS AD III na cidade João Pessoa, PB. Revista Saúde Debate, 2018; 42 (4): 275-284.

5. BRASIL, Ministério da Saúde. Reforma psiquiátrica e política de saúde mental no Brasil. Brasília, DF: Secretaria de Atenção à Saúde; 2005; 56 p.

6. BRASIL, Ministério da Saúde. Educação em Saúde diretrizes. Brasília, DF: Fundação Nacional de Saúde; $2007 ; 70$ p.

7. BRASIL, Ministério da Saúde. Orientações para elaboração de projetos de construção, reforma e ampliação de CAPS e de UA. Brasília, DF: Secretaria de Atenção à Saúde; 2015; 46 p.

8. BOSQUE RM, et al. Satisfação dos trabalhadores, usuários e familiares dos Centros de Atenção Psicossocial Álcool e outras Drogas. Revista de Enfermagem UFPE, 2017; 11 (11): 4598-4606.

9. CARDOSO MPO, et al. Práticas de cuidado em saúde mental na voz dos usuários de um Centro de Atenção Psicossocial do estado do Pará. Revista Saúde Debate, 2016; 40 (109): 86- 89.

10. CRUZ MP, et al. Oficinas terapêuticas em saúde mental como instrumento de reabilitação psicossocial: percepção dos familiares. Revista de Enfermagem, 2016; 10 (11): 3996 -3400.

11. DUTRA VFD, et al. Mediar a autonomia: um cuidado essencial em saúde mental. Escola Anne Nery Revista de Enfermagem, 2017; 21 (3): 1-8.

12. FALKENBERG MB, et al. Educação em saúde e educação na saúde: conceitos e implicações para a saúde coletiva. Revista Ciência e Saúde Coletiva, 2014; 19 (3): 847-852.

13. KAMI MTM, et al. Trabalho no consultório de rua: uso do software IRAMUTEQ no apoio à pesquisa qualitativa. Escola Anna Nery Revista de Enfermagem, 2016; 20 (3).

14. OLIVEIRA VC, et al. Perfil sociodemográfico e clínico de pessoas atendidas em um CAPS AD do sul do Brasil. Revista Baiana Enfermagem, 2017; 31 (1): 1-12.

15. PEIXOTO C, et al. Impacto do perfil clínico e sociodemográfico na adesão ao tratamento de pacientes de um Centro de Atenção Psicossocial a Usuários de Álcool e Drogas (CAPSad). Jornal Brasileiro de Psiquiatria, 2010; 59 (4): 317-321.

16. PFLUCK NAD. Práticas educativas em saúde: a experiência de um grupo educativo para usuários do Centro de Atenção Psicossocial- Álcool e outras drogas (CAPS AD) de Gravataí. Monografia (Especialização em psicologia clínica) - Instituto de Psicologia, Universidade Federal do Rio Grande do Sul. Porto Alegre, 2011; 54 p.

17. SCHLICKANN DS, et al. Oficina culinária como estratégia no cuidado em saúde mental. In: Anais do 2. Congresso Brasileiro Interdisciplinar de Promoção da Saúde; 2016 out 18-20; Rio Grande do Sul, Brasil. Rio Grande do Sul: Universidade de Santa Cruz do Sul; 2016.

18. SILVA E SILVA DL, et al. Perfil sociodemográfico e epidemiológico dos usuários de um Centro de Atenção Psicossocial Álcool e outras Drogas. Revista de Enfermagem e Atenção à Saúde, 2017; 6 (1): 67-79.

19. SILVA PPC, et al. Práticas corporais no Centro de Atenção Psicossocial de Álcool e Droga: a percepção dos usuários. Revista Brasileira Ciência e Esporte, 2019; 41 (1): 3-9.

20. STEILL EPS, et al. Os processos para a implantação de uma oficina terapêutica dentro do Centro de Atenção Psicossocial. Revista Brasileira de Pesquisa em Saúde, Vitória, 2017; 19 (1): 6-10.

21. VELÔSO TMG, EULÁLIO MC. Saúde Mental: saberes e fazeres. Editora da Universidade Estadual da Paraíba; $2016 ; 352 \mathrm{p}$. 